\title{
Vibration Modes and the Dynamic Behaviour of a Hydraulic Plunger Pump
}

\author{
Tianxiao Zhang and Nong Zhang \\ School of Electrical, Mechanical and Mechatronic Systems, University of Technology Sydney, 15 Broadway, Ultimo, NSW 2007, Australia \\ Correspondence should be addressed to Tianxiao Zhang; xiaozhang717@126.com
}

Received 19 August 2015; Revised 19 November 2015; Accepted 22 November 2015

Academic Editor: Vadim V. Silberschmidt

Copyright ( 92016 T. Zhang and N. Zhang. This is an open access article distributed under the Creative Commons Attribution License, which permits unrestricted use, distribution, and reproduction in any medium, provided the original work is properly cited.

\begin{abstract}
Mechanical vibrations and flow fluctuation give rise to complex interactive vibration mechanisms in hydraulic pumps. The working conditions for a hydraulic pump are therefore required to be improved in the design stage or as early as possible. Considering the structural features, parameters, and operating environment of a hydraulic plunger pump, the vibration modes for two-degree-offreedom system were established by using vibration theory and hydraulic technology. Afterwards, the analytical form of the natural frequency and the numerical solution of the steady-state response were deduced for a hydraulic plunger pump. Then, a method for the vibration analysis of a hydraulic pump was proposed. Finally, the dynamic responses of a hydraulic plunger pump are obtained through numerical simulation.
\end{abstract}

\section{Introduction}

Since there have been increasingly higher requirements imposed on the engineering quality, the accuracy, and reliability of products, it is an urgent task to study and solve various vibration problems existing in industrial machinery. Due to increasing system complexity, rapid operations, and improved accuracy of mechanical devices, vibration is a serious issue. Therefore, both the static strength effect and the dynamic force effect must be taken into account while designing mechanical devices [1-3].

Many studies have been performed to analyse the dynamic performance of hydraulic components and systems. To prevent hydraulic systems from breaking down, analysis of their vibration is required. An effective method of reducing vibration and noise needs to be developed: its aim is to improve the performance of hydraulic devices and thus reduce the vibration and noise in hydraulic systems. Vibration and noise arise from the interaction between solids and the fluid here. Fluid-structure interaction (FSI) not only reflects the essence of vibration noise in hydraulic system but also is the leitmotif for research in the field. Fluid-structure interaction would be caused between solid and liquid under different conditions. The field of hydraulic model and vibration has been discussed in some research [4-7]. A block diagram for the vibration analysis of a hydraulic component and system is shown in Figure 1.

A hydraulic pump is the main vibration and noise source in a hydraulic system, and its working state determines the safe operation of hydraulic components therein. Therefore, it can be seen that mechanical vibrations and flow fluctuations not only affect the engineering quality but also reduce the lifespan of hydraulic components and systems, generate noise pollution, and even cause damage. Furthermore, they may give rise to accidents. Studies relating the vibration of hydraulic pump mainly focus on the analysis of test data and the reduction in vibration and noise [8-16], and quantitative simulations have also been conducted to analyse the vibrations thereof. The vibration analysis of a hydraulic pump is beneficial for controlling noise and vibration.

Influenced by the design, structure, and operating environment of a hydraulic plunger pump as well as the inherent characteristic curve, flow pulsation is bound to be generated. Flow and pressure pulsation are two of the leading reasons for noise and vibration being generated by a hydraulic pump. This paper gave full considerations to the vibration problems caused by flow and pressure pulsation in a hydraulic plunger pump and converted the simplified formula into an 


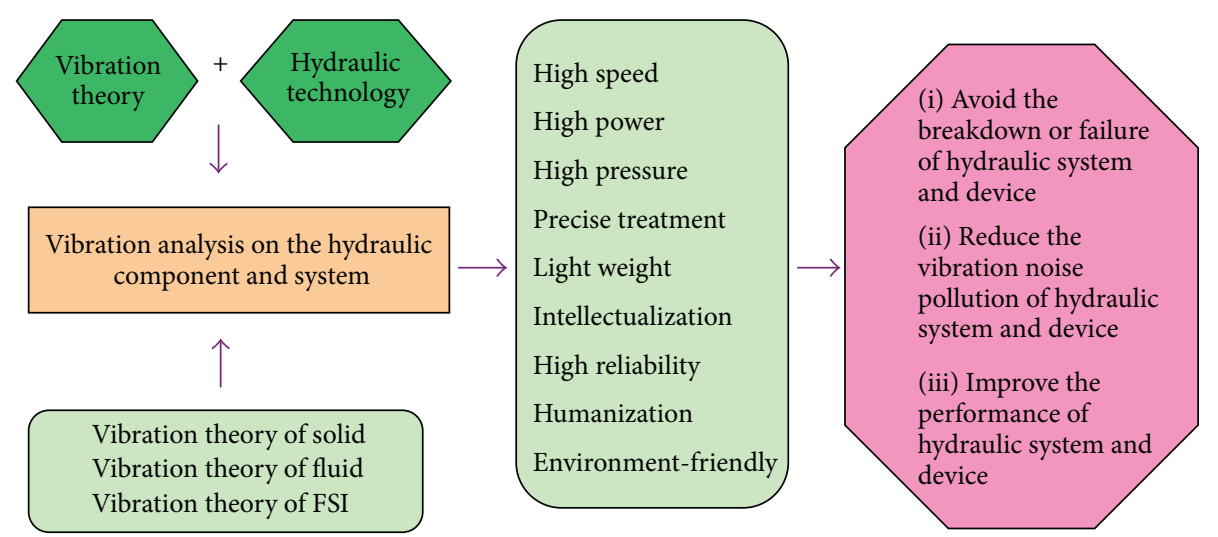

FIgURE 1: Vibration analysis: hydraulic component and system.

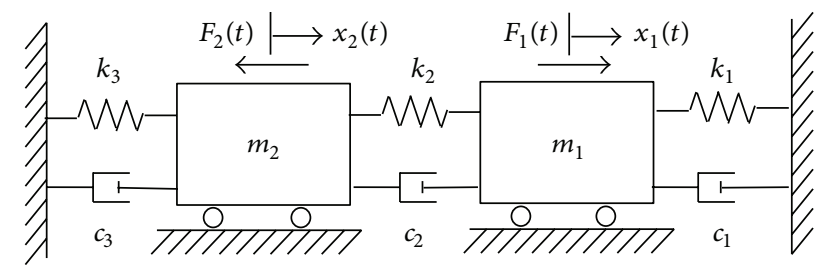

FIGURE 2: Vibration model for the two degrees of freedom of a hydraulic plunger pump.

excitation describing the vibration system of a plunger pump. In addition, a vibration mode for the two degrees of freedom in a hydraulic plunger pump was made available for practical calculation and was established to estimate the vibration of a hydraulic plunger pump under specific working conditions. Meanwhile, the model reveals the basic mechanisms of vibration and noise in a hydraulic plunger pump. By conducting the dynamic analysis using the proposed approach, a better understanding of hydraulic plunger pumps can be obtained.

\section{Vibration Model for \\ Two-Degree-of-Freedom System for a Hydraulic Plunger Pump}

2.1. Vibration Model of Plunger Pump. The vibration model for the two degrees of freedom of a hydraulic plunger pump was developed based on the data and conditions regarding the structural features, parameters, variables, constraints, operational states, and flow pulsation. The model is shown in Figure 2. It was assumed that the mass of the cylinder block of the hydraulic plunger pump was $m_{1}(\mathrm{~kg})$, and the masses of its retainer plate, seven plungers, and sliding shoes were merged and represented by $m_{2}(\mathrm{~kg})$. The connection stiffness and the damping between the cylinder block and port plate were, respectively, set to $k_{1}(\mathrm{~N} / \mathrm{m})$ and $c_{1}(\mathrm{~N} \cdot \mathrm{s} / \mathrm{m})$. The cylinder and retainer plate were connected by a central spring whose stiffness and damping were $k_{2}(\mathrm{~N} / \mathrm{m})$ and $c_{2}$ $(\mathrm{N} \cdot \mathrm{s} / \mathrm{m})$, respectively. The sliding shoes were connected with an angle plate with a connection stiffness and damping of $k_{3}$ $(\mathrm{N} / \mathrm{m})$ and $c_{3}(\mathrm{~N} \cdot \mathrm{s} / \mathrm{m})$, respectively.
According to the proposed vibration model, the positive directions of the acceleration and excitation were determined, which were in accordance with the positive direction of the coordinate axes. The deduced vibration differential equation is

$$
\begin{gathered}
{\left[\begin{array}{cc}
m_{1} & 0 \\
0 & m_{2}
\end{array}\right]\left\{\begin{array}{l}
\ddot{x}_{1} \\
\ddot{x}_{2}
\end{array}\right\}+\left[\begin{array}{cc}
c_{1}+c_{2} & -c_{2} \\
-c_{2} & c_{2}+c_{3}
\end{array}\right]\left\{\begin{array}{l}
\dot{x}_{1} \\
\dot{x}_{2}
\end{array}\right\}} \\
+\left[\begin{array}{cc}
k_{1}+k_{2} & -k_{2} \\
-k_{2} & k_{2}+k_{3}
\end{array}\right]\left\{\begin{array}{l}
x_{1} \\
x_{2}
\end{array}\right\}=\left\{\begin{array}{c}
F_{1}(t) \\
-F_{2}(t)
\end{array}\right\},
\end{gathered}
$$

where the constant matrices including $\mathbf{M}=[m], \mathbf{C}=[c]$, and $\mathbf{K}=[k]$, which are constituted by coefficient matrices, are, respectively, the mass matrix, damping matrix, and stiffness matrix: the vector $\mathbf{x}=\{x\}$ is the displacement vector.

2.2. Flow Pulsation. One of the most influential factors in the vibration model of hydraulic plunger pump is the value of input vibration excitation; because flow pulsation is the source of pressure pulsation, flow pulsation has to be analysed to study pressure pulsation. Flow pulsation refers to the instantaneous flow variation when a hydraulic pump is running. When a hydraulic pump keeps operating continuously, the ever-changing sealing volume is expected to be generated in a majority of hydraulic pumps. Meanwhile, the instantaneous flow changes repeatedly, and some instantaneous, nonconstant flow may generate flow pulsation. The instantaneous actual flow of a hydraulic plunger pump may be given as follows.

When $0 \leq \varphi \leq \alpha$ and $z_{0}=(z+1) / 2$,

$$
\begin{aligned}
q_{s 1} & =A R \omega \eta_{V} \tan \gamma \frac{\cos (\alpha / 2-\varphi)}{2 \sin (\alpha / 2)} \\
& =\frac{\pi d^{2} R \omega \eta_{V} \tan \gamma}{8 \sin (\alpha / 2)} \cos (\alpha / 2-\varphi) .
\end{aligned}
$$

When $\alpha \leq \varphi \leq 2 \alpha$ and $z_{0}=(z-1) / 2$,

$$
\begin{aligned}
q_{s 2} & =A R \omega \eta_{V} \tan \gamma \frac{\cos (3 \alpha / 2-\varphi)}{2 \sin (\alpha / 2)} \\
& =\frac{\pi d^{2} R \omega \eta_{V} \tan \gamma}{8 \sin (\alpha / 2)} \cos (3 \alpha / 2-\varphi),
\end{aligned}
$$


where $A$ represents the cross-sectional area $\left(\mathrm{m}^{2}\right)$ of the plunger; $R$ is the radius $(\mathrm{m})$ of the central circle of the plunger, and $d$ indicates the diameter $(\mathrm{m})$ of the plunger; $\gamma$ denotes the inclination angle (rad) of the angle plate, $z$ is the number of the plungers, and $\omega$ is the angular velocity $(\mathrm{rad} / \mathrm{s})$ of the oil cylinder block; $n$ is the rotational speed (rpm) of the plunger pump, $\eta_{V}$ is the volumetric efficiency, and $\alpha$ is the half included angle (rad) between two adjacent plungers and $\alpha=\pi / z ; \varphi$ represents the angular displacement (rad) of the cylinder block, and $\varphi=\omega t$. Let

$$
K_{q}=\frac{\pi d^{2} R \omega \eta_{V} \tan \gamma}{8 \sin (\alpha / 2)}=\frac{\pi d^{2} R \omega \eta_{V} \tan \gamma}{8 \sin (\pi / 2 z)} .
$$

Then

$$
\begin{aligned}
& q_{s 1}=K_{q} \cos \left(\frac{\alpha}{2}-\varphi\right), \\
& q_{s 2}=K_{q} \cos \left(\frac{3 \alpha}{2}-\varphi\right) .
\end{aligned}
$$

Converting the instantaneous actual flow into a function of time $t(s)$, then

$$
\begin{aligned}
& q_{s 1}=K_{q} \cos \left(\omega t-\frac{\pi}{2 z}\right) \quad \text { in case } 0 \leq t \leq \frac{\pi}{z \omega}, \\
& q_{s 2}=K_{q} \cos \left(\omega t-\frac{3 \pi}{2 z}\right) \quad \text { in case } \frac{\pi}{z \omega} \leq t \leq \frac{2 \pi}{z \omega},
\end{aligned}
$$

where $q_{s 1}$ and $q_{s 2}$ represent the instantaneous flow through the plunger pump. It can be seen that when $\varphi=\alpha / 2$ and $\varphi=$ $3 \alpha / 2$ (namely, $\varphi=\pi / 2 z$ and $\varphi=3 \pi / 2 z$ ), instantaneous flow was at a maximum, $q_{s \max }$; when $\varphi=0$ and $\varphi=\alpha$ (namely, $\varphi=$ 0 and $\varphi=\pi / z)$, instantaneous flow was at a minimum, $q_{s \min }$. Therefore, the flow pulsation coefficient $\delta_{q}$ can be defined as follows:

$$
\delta_{q}=\frac{q_{s \max }-q_{s \min }}{q_{t}},
$$

where $q_{t}$ is the theoretical mean flow. When the cylinder block of the plunger pump rotates through one complete revolution, each plunger moves back and forth once in a cycle of oil adsorption and extrusion. Therefore, the theoretical mean flow $q_{t}$ and the actual flow $q$ can be presented as

$$
\begin{aligned}
q_{t} & =2 A z n R \tan \gamma=15 z \omega d^{2} R \tan \gamma, \\
q & =2 A z n R \eta_{V} \tan \gamma=15 z \omega d^{2} R \eta_{V} \tan \gamma .
\end{aligned}
$$

\subsection{Pressure Pulsation. Flow pulsation inevitably gives rise to} pressure pulsation, which indicates that the flow and pressure output from the hydraulic plunger pump change with time. Therefore, the flow and pressure output by hydraulic plunger pump are not necessarily stable. The change in the volume of a hydraulic plunger pump always results in fluctuations in output pressure and fluid flow, leading to the generation of noise and vibration. According to the fundamental principles of fluid dynamics, the change of the flow in the closed cavity between the plunger and cylinder block of a hydraulic plunger pump inevitably leads to a pressure change. With respect to a compressible flow, its instantaneous pressure can be represented by the following formulas.

When $0 \leq t \leq \pi / z \omega$,

$$
\begin{aligned}
p_{s 1} & =\frac{E_{q}}{7 V_{q}} \int_{0}^{t} q_{s 1} \mathrm{~d} t=\frac{E_{q} K_{q}}{V_{q}} \int_{0}^{t} \cos \left(\omega t-\frac{\pi}{2 z}\right) \mathrm{d} t \\
& =\frac{E_{q} K_{q}}{7 V_{q} \omega}\left[\sin \left(\omega t-\frac{\pi}{2 z}\right)+\sin \left(\frac{\pi}{2 z}\right)\right] .
\end{aligned}
$$

When $\pi / z \omega \leq t \leq 2 \pi / z \omega$,

$$
\begin{aligned}
p_{s 2} & =\frac{E_{q}}{7 V_{q}} \int_{\pi / z \omega}^{t} q_{s 2} \mathrm{~d} t \\
& =\frac{E_{q} K_{q}}{7 V_{q}}\left[\int_{\pi / z \omega}^{t} \cos \left(\omega t-\frac{3 \pi}{2 z}\right) \mathrm{d} t\right] \\
& =\frac{E_{q} K_{q}}{7 V_{q} \omega}\left[\sin \left(\omega t-\frac{3 \pi}{2 z}\right)+\sin \left(\frac{\pi}{2 z}\right)\right],
\end{aligned}
$$

where $E_{q}$ represents the elastic modulus of the flow $(\mathrm{Pa})$ and the volume of the closed cavity for a single plunger is $V_{q}=$ $A_{d} s=\left(\pi d^{2} / 2\right) R \tan \gamma$.

2.4. Fourier Series Simulation. The flow and pressure pulsation in a hydraulic plunger pump are a nonharmonic periodic function, which can be represented by a harmonic Fourier series. Thereby, the dynamic response problem corresponding to the harmonic excitation of its Fourier series can be solved. The periodic excitation function in an interval of $\left[e_{1}, e_{2}\right]$ is unfolded as the Fourier series; namely,

$$
\begin{aligned}
& F(x)=\frac{a_{0}}{2}+\sum_{j=1}^{\infty}\left(a_{j} \cos \frac{j \pi\left(2 x-e_{1}-e_{2}\right)}{e_{2}-e_{1}}\right. \\
& \left.+b_{j} \sin \frac{j \pi\left(2 x-e_{1}-e_{2}\right)}{e_{2}-e_{1}}\right)=\frac{a_{0}}{2} \\
& +\sum_{j=1}^{\infty}\left[a_{j} \cos j(z \omega x-\pi)+b_{j} \sin j(z \omega x-\pi)\right],
\end{aligned}
$$

where $e_{1}=0$ and $e_{2}=2 \pi / z \omega$. Equation (9) shows that a complex periodic excitation function can be decomposed into the superposition of a series of harmonic functions. The coefficients of Fourier series $a_{0}, a_{j}$, and $b_{j}$ can be determined as follows:

$$
\begin{aligned}
a_{j} & =\frac{2}{e_{2}-e_{1}} \int_{e_{1}}^{e_{2}} F(t) \cos \frac{j \pi\left(2 t-e_{1}-e_{2}\right)}{e_{2}-e_{1}} \mathrm{~d} t \\
& =\frac{z \omega}{\pi} \int_{0}^{2 \pi / z \omega} F(t) \cos j(z \omega t-\pi) \mathrm{d} t
\end{aligned}
$$

$$
(j=0,1,2,3, \ldots)
$$




$$
\begin{aligned}
b_{j}= & \frac{2}{e_{2}-e_{1}} \int_{e_{1}}^{e_{2}} F(t) \sin \frac{j \pi\left(2 t-e_{1}-e_{2}\right)}{e_{2}-e_{1}} \mathrm{~d} t \\
=\frac{z \omega}{\pi} \int_{0}^{2 \pi / z \omega} F(t) \sin j(z \omega t-\pi) \mathrm{d} t & \\
& (j=1,2,3, \ldots) .
\end{aligned}
$$

The function $F(x)$ can be represented by Fourier series as long as the defined integrations of $a_{j}$ and $b_{j}$ actually exist. If $F(x)$ cannot be represented by means of function, it can be calculated through approximate calculation. The periodic pressure pulsation indicated by formulas (8a) and (8b) is unfolded as the Fourier series, and the coefficient of Fourier series is

$$
\begin{aligned}
& a_{0}=\frac{2 E_{q} K_{q}}{7 V_{q} \omega^{2}} \sin \left(\frac{\pi}{2 z}\right), \\
& a_{j}=\frac{z \omega}{\pi} \frac{E_{q} K_{q}}{7 V_{q} \omega}\left[\int_{0}^{\pi / z \omega}\left[\sin \left(\omega t-\frac{\pi}{2 z}\right)+\sin \left(\frac{\pi}{2 z}\right)\right]\right. \\
& \cdot \cos j(z \omega t-\pi) \mathrm{d} t]+\frac{z \omega}{\pi} \frac{E_{q} K_{q}}{7 V_{q} \omega} \\
& \cdot \int_{\pi / z \omega}^{2 \pi / z \omega}\left[\sin \left(\omega t-\frac{3 \pi}{2 z}\right)+\sin \left(\frac{\pi}{2 z}\right)\right] \\
& \cdot \cos j(z \omega t-\pi) \mathrm{d} t=0 \quad(j=1,2,3, \ldots), \\
& b_{j}=\frac{z \omega}{\pi} \frac{E_{q} K_{q}}{7 V_{q} \omega} \int_{0}^{\pi / z \omega}\left[\sin \left(\omega t-\frac{\pi}{2 z}\right)+\sin \left(\frac{\pi}{2 z}\right)\right] \\
& \cdot \sin j(z \omega t-\pi) \mathrm{d} t+\frac{z \omega}{\pi} \frac{E_{q} K_{q}}{7 V_{q} \omega} \\
& \cdot \int_{\pi / z \omega}^{2 \pi / z \omega}\left[\sin \left(\omega t-\frac{3 \pi}{2 z}\right)+\sin \left(\frac{\pi}{2 z}\right)\right] \\
& \cdot \sin j(z \omega t-\pi) \mathrm{d} t=\frac{z \omega}{\pi} \frac{E_{q} K_{q}}{7 V_{q} \omega}\left\{-\frac{1}{(1+j z) \omega}\right. \\
& \left.\cdot \sin \left(\frac{\pi}{2 z}+j \pi\right)+\frac{1}{(1-j z) \omega} \sin \left(\frac{\pi}{2 z}-j \pi\right)\right\} \\
& (j=1,2,3, \ldots) \text {. }
\end{aligned}
$$

Therefore, the quantity of pressure pulsation induced by the quantity of flow pulsation can be expressed as

$$
p_{s}(t)=\frac{a_{0}}{2}+\sum_{j=1}^{\infty} b_{j} \sin j(z \omega t-\pi) \quad(j=1,2,3, \ldots),
$$

where $p_{s}$ is the quantity of pressure pulsation of the plunger pump. The sum of pressure pulsation and mean pressure expresses the pressure work in pump; that is, $p(t)=p_{0}+p_{s}(t)$. That means the pressure work in pump is always greater than zero. According to (12), the multiseries pressure pulsation function can be simulated using a Fourier series, and Figure 3 shows the pressure pulsation function as $j(j=1,2, \ldots, 10)$ with its different values.
From Figure 3, the quantity of pressure pulsation change reciprocated at mean pressure port, positive value of vertical axis expresses the direction of pulsation quantity which is the same as $x$-axis, negative value of vertical axis expresses the direction of pulsation quantity which is opposite to $x$-axis, and the pulsation quantity function is periodical change in value and direction. Therefore, as long as relevant conditions are satisfied, all periodic functions can be described as harmonic, according to Fourier series; the complicated quantity of pressure pulsation function is expressed as harmonic function, convergent Fourier series: this solves the response problem of harmonic excitation and caused piston pump to move circularly.

2.5. System Excitation. The excitation of the vibration system for the two-degree-of-freedom system of a hydraulic plunger pump caused by flow and pressure pulsation is given by

$$
F_{1}(t)=p_{s} A_{C}
$$

where $A_{C}$ represents the cross-sectional area of the cylinder hole of the cylinder block, $A_{C}=7(\pi / 4) d^{2}$, and $d$ is the diameter of piston. Consider

$$
F_{2}(t)=7 p_{s} A_{P}
$$

where $A_{P}$ is the cross-sectional area of a single plunger and here $A_{C} \approx 7 A_{P}$; therefore, the excitation $F_{2}(t)$ is considered to be equal to $-F_{1}(t)$, where $F_{1}(t)$ and $F_{2}(t)$ are a pair of action and counteraction forces with the same magnitude but acting in opposite directions.

\section{Vibration of the Two-Degree-of-Freedom System of a Hydraulic Plunger Pump}

3.1. The Natural Frequency and Modes of Vibration of the System. As seen from (1), matrices $\mathbf{M}=[m], \mathbf{C}=[c]$, and $\mathbf{K}=[k]$ are symmetrical, and the matrix elements are, respectively, listed as follows:

$$
\begin{aligned}
& m_{11}=m_{1}, \\
& m_{12}=m_{21}=0, \\
& m_{22}=m_{2}, \\
& c_{11}=c_{1}+c_{2}, \\
& c_{12}=c_{21}=-c_{2}, \\
& c_{22}=c_{2}+c_{3}, \\
& k_{11}=k_{1}+k_{2}, \\
& k_{12}=k_{21}=-k_{2}, \\
& k_{22}=k_{2}+k_{3} .
\end{aligned}
$$




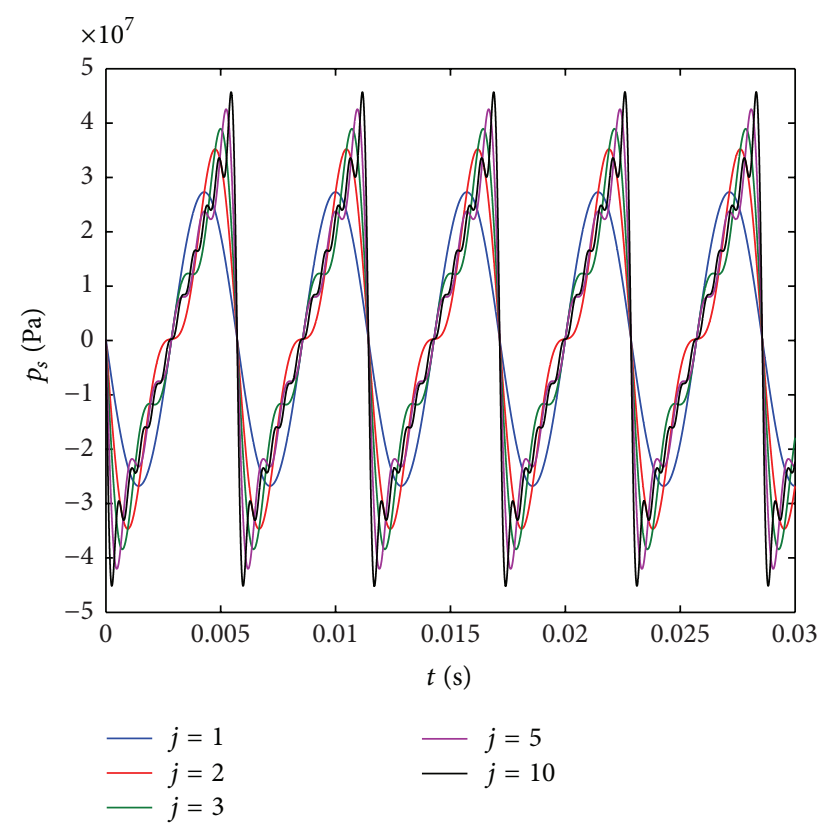

Figure 3: Pressure pulsation $p_{s}(\mathrm{~Pa})$.

To study the inherent characteristics of a hydraulic plunger pump, the deduced characteristic equation, or frequency equation, of the two-degree-of-freedom system of a hydraulic plunger pump may be given as follows:

$$
\begin{aligned}
\Delta\left(\omega^{2}\right)= & m_{1} m_{2} \omega^{4}-\left(m_{1} k_{22}+m_{2} k_{11}\right) \omega^{2}+k_{11} k_{22} \\
& -k_{12}^{2}=0 .
\end{aligned}
$$

Equation (15) is a quadratic equation in $\omega^{2}$, and its roots are

$$
\begin{aligned}
\omega_{1}^{2}= & \frac{1}{2} \frac{m_{1} k_{22}+m_{2} k_{11}}{m_{1} m_{2}} \\
& \mp \frac{1}{2} \sqrt{\left(\frac{m_{1} k_{22}+m_{2} k_{11}}{m_{1} m_{2}}\right)^{2}-4 \frac{k_{11} k_{22}-k_{12}^{2}}{m_{1} m_{2}} .}
\end{aligned}
$$

Since $k_{11} k_{22}=\left(k_{1}+k_{2}\right)\left(k_{2}+k_{3}\right)>k_{2}^{2}=k_{12}^{2}$, as seen in (16), the value of the item following the $\mp$ sign was smaller than that to the left of it, and thus $\omega_{1}^{2}$ and $\omega_{2}^{2}$ are positive numbers. Therefore, two positive real roots of the characteristic equation in (15) can be obtained, namely, the two natural frequencies $\omega_{1}$ and $\omega_{2}$ of the system.

$u_{1}^{(1)}$ and $u_{2}^{(1)}$ are used to represent the amplitudes corresponding to $\omega_{1}$, and $u_{1}^{(2)}$ and $u_{2}^{(2)}$ are the amplitudes corresponding to $\omega_{2}$. The homogeneous differential equation can only determine the ratios of $u_{2}^{(1)} / u_{1}^{(1)}$ and $u_{2}^{(2)} / u_{1}^{(2)}$. The deduced amplitude ratios are shown as follows:

$$
\begin{aligned}
& r_{1}=\frac{u_{2}^{(1)}}{u_{1}^{(1)}}=-\frac{k_{11}-\omega_{1}^{2} m_{1}}{k_{12}}=-\frac{k_{12}}{k_{22}-\omega_{1}^{2} m_{2}}, \\
& r_{2}=\frac{u_{2}^{(2)}}{u_{1}^{(2)}}=-\frac{k_{11}-\omega_{2}^{2} m_{1}}{k_{12}}=-\frac{k_{12}}{k_{22}-\omega_{2}^{2} m_{2}} .
\end{aligned}
$$

Therefore, the constants in pairs such as $u_{1}^{(1)}$ and $u_{2}^{(1)}$ and $u_{1}^{(2)}$ and $u_{2}^{(2)}$ can determine the natural vibration modes or main vibration mode displayed by the system when the system conducts synchronous harmonic motion, respectively, at frequencies of $\omega_{1}$ and $\omega_{2}$. These constants can be expressed by the following matrices:

$$
\begin{aligned}
& \mathbf{u}^{(1)}=\left\{\begin{array}{l}
u_{1}^{(1)} \\
u_{2}^{(1)}
\end{array}\right\}=u_{1}^{(1)}\left\{\begin{array}{l}
1 \\
r_{1}
\end{array}\right\}, \\
& \mathbf{u}^{(2)}=\left\{\begin{array}{l}
u_{1}^{(2)} \\
u_{2}^{(2)}
\end{array}\right\}=u_{1}^{(2)}\left\{\begin{array}{l}
1 \\
r_{2}
\end{array}\right\} .
\end{aligned}
$$

There are two natural frequencies in this system, and correspondingly there are two natural vibration modes. The lower frequency $\omega_{1}$ is the first-order natural frequency or, simply, the fundamental frequency, while the higher frequency $\omega_{2}$ is the second-order natural frequency. The corresponding vibration modes $\mathbf{u}^{(1)}$ and $\mathbf{u}^{(2)}$ are, respectively, the first-order and second-order natural vibration modes.

3.2. Dynamic Response of the Pump System. With the improvement and development of computer software, hardware, and technology, almost all engineering problems can be simulated quantitatively with high precision. Numerical simulation involves the solution of a mathematical problem whose exact solution is hard to find in practical engineering. The numerical simulation applied to mechanical vibration problems must discrete the time history for a dynamic response within the time domain so as to discretize the differential equation of motion and numerical equations at different moments. Meanwhile, the speed and acceleration at a given time are described by the combination of the displacements at adjacent time steps. As a result, the differential equation of motion of the system is converted into algebraic equations at discrete time steps. Then, the values corresponding to a series of discrete times are obtained by numerical integration of the differential equation of motion of the coupling system. There are many commonly used approaches for finding the dynamic response of such systems, including (1) central difference, (2) Houbolt, (3) Wilson- $\theta$, and (4) Newmark- $\beta$.

\section{A Numerical Example}

The mass of the cylinder block of a certain hydraulic plunger pump was $m_{1}=2.7 \mathrm{~kg}$, and the masses of the retainer plate, seven plungers, and its sliding shoes were merged together as $m_{2}=1.8825 \mathrm{~kg}$. Suppose that the connection stiffness and damping between the cylinder block and the port plate were, respectively, $k_{1}=3.6 \times 10^{7} \mathrm{~N} / \mathrm{m}$ and $c_{1}=329.0 \mathrm{~N} \cdot \mathrm{s} / \mathrm{m}$, and the stiffness and damping between the cylinder and the retainer plate were $k_{2}=5.0 \times 10^{5} \mathrm{~N} / \mathrm{m}$ and $c_{2}=223.0 \mathrm{~N} \cdot \mathrm{s} / \mathrm{m}$, respectively; the stiffness and damping between the sliding shoes and the swash plate were, respectively, $k_{3}=7.7 \times$ $10^{7} \mathrm{~N} / \mathrm{m}$ and $c_{3}=490.0 \mathrm{~N} \cdot \mathrm{s} / \mathrm{m}$. The inclination angle of the swash plate was $\gamma=19.5^{\circ}$, the radius of the central circle of the plunger was $R=0.285 \mathrm{~m}$, the diameter was $d=0.019 \mathrm{~m}$, 
and number of the plungers was $z=7$. Meanwhile, the rate of rotation of the plunger pump was $n=1500 \mathrm{rpm}$. The elastic modulus of the flow and the volumetric efficiency of the plunger pump were, respectively, $E_{q}=1.226 \times 10^{3} \mathrm{MPa}$ and $\eta_{V}=0.95$. This research attempted to obtain the natural frequency and vibration modes of the system as well as its vibration response.

According to the known conditions, here we have

$$
\begin{aligned}
& k_{11}=k_{1}+k_{2}, \\
& k_{12}=k_{21}=-k_{2}, \\
& k_{22}=k_{2}+k_{3} .
\end{aligned}
$$

By substituting the above into (16), the natural frequency could be obtained:

$$
\begin{aligned}
& \omega_{1}=3676 \frac{1}{\mathrm{~s}}, \\
& \omega_{2}=6416 \frac{1}{\mathrm{~s}} .
\end{aligned}
$$

The values of $r_{1}$ and $r_{2}$ can be obtained by substituting $\omega_{1}^{2}$ and $\omega_{2}^{2}$ into (17a) and (17b):

$$
\begin{aligned}
& r_{1}=0.0096, \\
& r_{2}=-149.3203 .
\end{aligned}
$$

The natural modes of vibration for the system can be calculated according to (18a) and (18b):

$$
\begin{aligned}
& \mathbf{u}^{(1)}=\left\{\begin{array}{c}
1 \\
0.0096
\end{array}\right\}, \\
& \mathbf{u}^{(2)}=\left\{\begin{array}{c}
1 \\
-149.3203
\end{array}\right\} .
\end{aligned}
$$

Figure 4 shows the two natural modes of vibration: in the first-order vibration mode, the two masses move forward with an amplitude ratio of 1:0.0119; in the second-order vibration mode, the two masses move adversely with an amplitude ratio of $1: 120.9773$. It is noted that there is a nodal point of zero displacement in the second-order natural mode of vibration.

Newmark- $\beta$ method was applied to the vibration model here, and the values of $\beta$ and $\delta$ were taken, respectively, as $\beta=1 / 4$ and $\delta=1 / 2$. The displacement $x_{1}(\mathrm{~m})$ of mass $m_{1}$ and displacement $x_{2}(\mathrm{~m})$ of mass $m_{2}$ over time were as shown in Figure 5.

As discovered during calculation, owing to the unstable initial running stage of the hydraulic plunger pump and the superposition of transient and steady-state vibrations, the system vibrated irregularly at large amplitude. However, the transient vibration gradually weakened and finally vanished after a period of time, and the system vibration reached a steady state. The amplitude of steady-state vibration of the plunger pump was acceptable in its smooth running stage. Meanwhile, compared with the retainer plate, seven plungers,
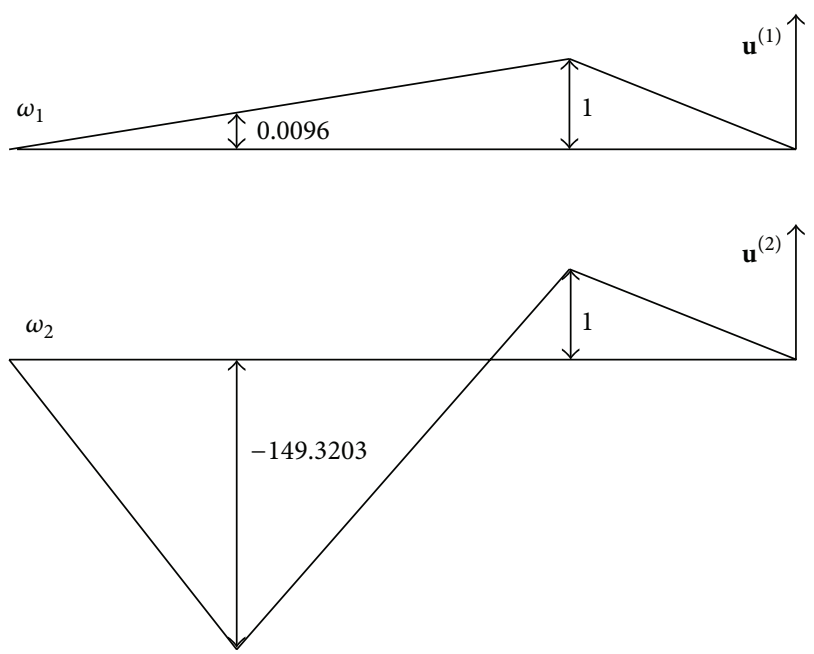

FIGURE 4: Natural mode of vibration of the two-degree-of-freedom system.

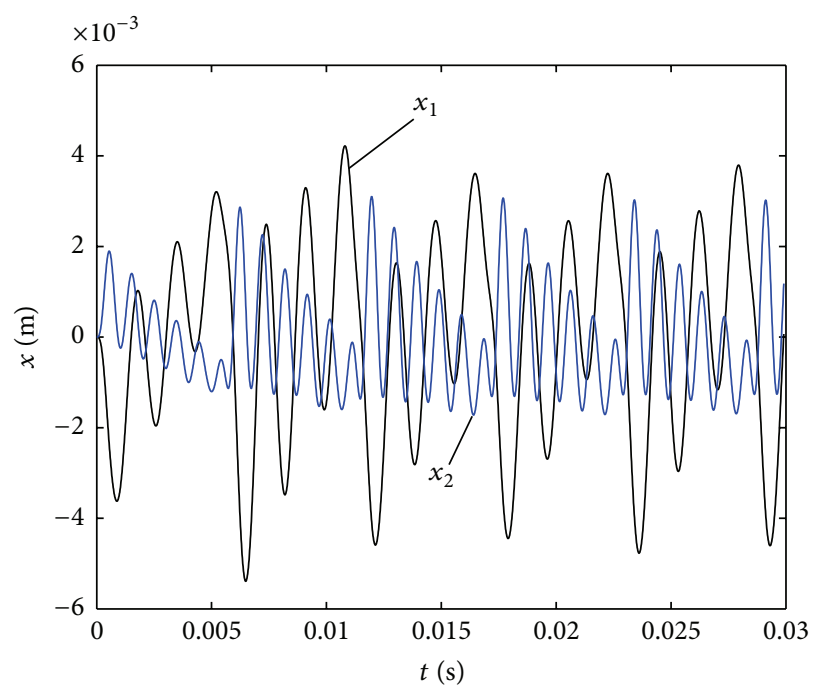

FIGURE 5: The response of the two-degree-of-freedom system with time $t$.

and sliding shoes, the amplitude of the cylinder block of the plunger pump is much greater. The numerical calculation results can provide quantitative theoretical support for the accurate design of a hydraulic plunger pump.

\section{Conclusions}

The mechanical vibration analysis and the dynamic design are key points during the engineering design of mechanical products and are crucial for producing products with the required dynamic characteristics. While conducting vibration analysis and dynamic design on a hydraulic plunger pump using the proposed approach, the actual design level and dynamic characteristics of the hydraulic plunger pump are expected to be improved. This can also prevent malfunctions and accidents caused by breakdowns. Based on using vibration theory and hydraulic technology, this research has developed a vibration 
model for the dynamic analysis of a two-degree-of-freedom hydraulic plunger pump system. Meanwhile, the inherent characteristics and dynamic response of the hydraulic plunger pump were studied and were used to estimate the vibration of a hydraulic plunger pump under regulated working conditions. In addition, the basic mechanisms of noise and vibration in a hydraulic plunger pump were revealed.

\section{Conflict of Interests}

The authors declare that there is no conflict of interests regarding the publication of this paper.

\section{Acknowledgments}

This research was supported by China Natural Science Foundation Project (Grant no. 51135003), the National Key Development Programme for Fundamental Research (973 Programme, Grant no. 2014CB046303), and Australian Research Council (ARC DP150102751).

\section{References}

[1] S. Timoshenko, D. H. Young, and W. Weaver Jr., Vibration Problems in Engineering, John Wiley \& Sons, New York, NY, USA, 4th edition, 1974.

[2] L. Meirovitch, Elements of Vibration Analysis, McGraw-Hill, New York, NY, USA, 1975.

[3] F. S. Tse, I. E. Morse, and R. T. Hinkle, Mechanical Vibrations Theory and Applications, Allyn and Bacon, Boston, Mass, USA, 1978.

[4] W. Sochacki, "Modelling and analysis of damped vibration in hydraulic cylinder," Mathematical and Computer Modelling of Dynamical Systems, vol. 21, no. 1, pp. 23-37, 2015.

[5] D. Cekus and B. Posiadala, "Vibration model and analysis of three-member telescopic boom with hydraulic cylinder for its radius change," International Journal of Bifurcation and Chaos, vol. 21, no. 10, pp. 2883-2892, 2011.

[6] A. Turnip, K.-S. Hong, and S. Park, "Modeling of a hydraulic engine mount for active pneumatic engine vibration control using the extended Kalman filter," Journal of Mechanical Science and Technology, vol. 23, no. 1, pp. 229-236, 2009.

[7] B. P. Bettig and R. P. S. Han, "Modeling the lateral vibration of hydraulic turbine-generator rotors," Journal of Vibration and Acoustics - Transactions of the ASME, vol. 121, no. 3, pp. 322327, 1999.

[8] I. K. Dulay, Fundamentals of Hydraulic Power Transmission, Elsevier Science Publishers, Amsterdam, The Netherlands, 1988.

[9] M. Noah, Hydraulic Control Systems, John Wiley \& Sons, Hoboken, NJ, USA, 2005.

[10] A. Akers, M. Gassman, and R. Smith, Hydraulic Power System Analysis, CRC Press, Boca Raton, Fla, USA, 2006.

[11] J. Houghtalen Robert, A. A. Osman, and H. C. Hwang Ned, Fundamentals of Hydraulic Engineering Systems, Prentice Hall, London, UK, 4th edition, 2009.

[12] P. Kumar, Hydraulic Machines: Fundamentals of Hydraulic Power Systems, CRC Press, Boca Raton, Fla, USA, 2012.

[13] R. T. Burton, P. R. Ukrainetz, P. N. Nikiforuk, and G. J. Schoenau, "Neural networks and hydraulic control-from simple to complex applications," Proceedings of the Institution of
Mechanical Engineers, Part I: Journal of Systems and Control Engineering, vol. 213, no. 5, pp. 349-358, 1999.

[14] R. Spence and J. Amaral-Teixeira, "Investigation into pressure pulsations in a centrifugal pump using numerical methods supported by industrial tests," Computers \& Fluids, vol. 37, no. 6, pp. 690-704, 2008.

[15] B. Zhang, B. Xu, C. L. Xia, and H. Y. Yang, "Modeling and simulation on axial piston pump based on virtual prototype technology," Chinese Journal of Mechanical Engineering, vol. 22, no. 1, pp. 84-90, 2009.

[16] B. Xu, J. Zhang, and H. Yang, "Simulation research on distribution method of axial piston pump utilizing pressure equalization mechanism," Proceedings of the Institution of Mechanical Engineers Part C: Journal of Mechanical Engineering Science, vol. 227, no. 3, pp. 459-469, 2013. 


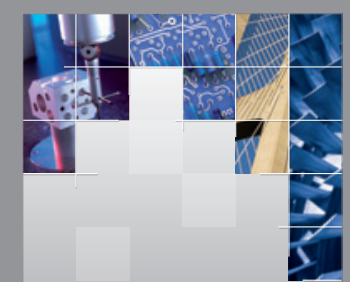

\section{Enfincering}
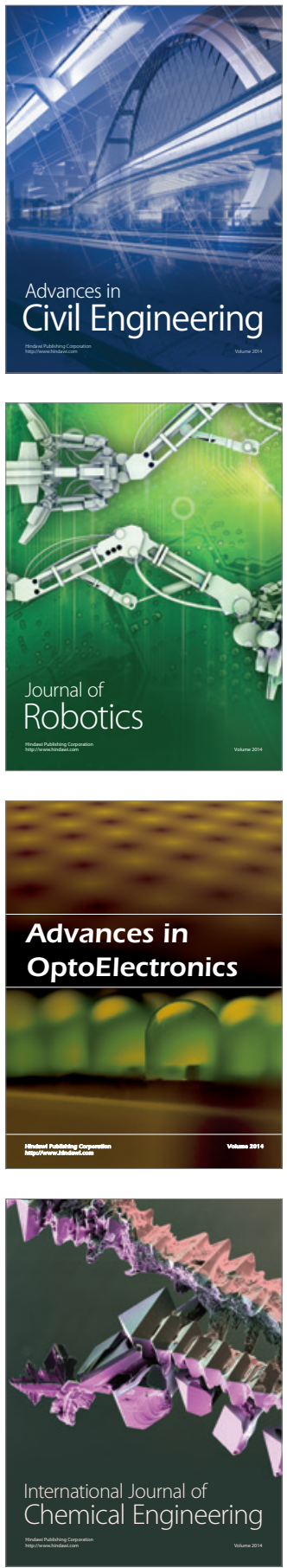

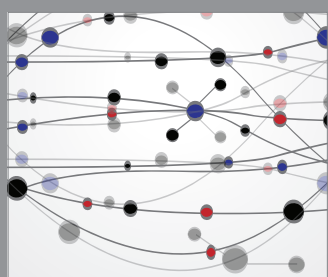

The Scientific World Journal

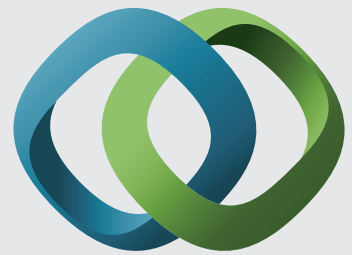

\section{Hindawi}

Submit your manuscripts at

http://www.hindawi.com
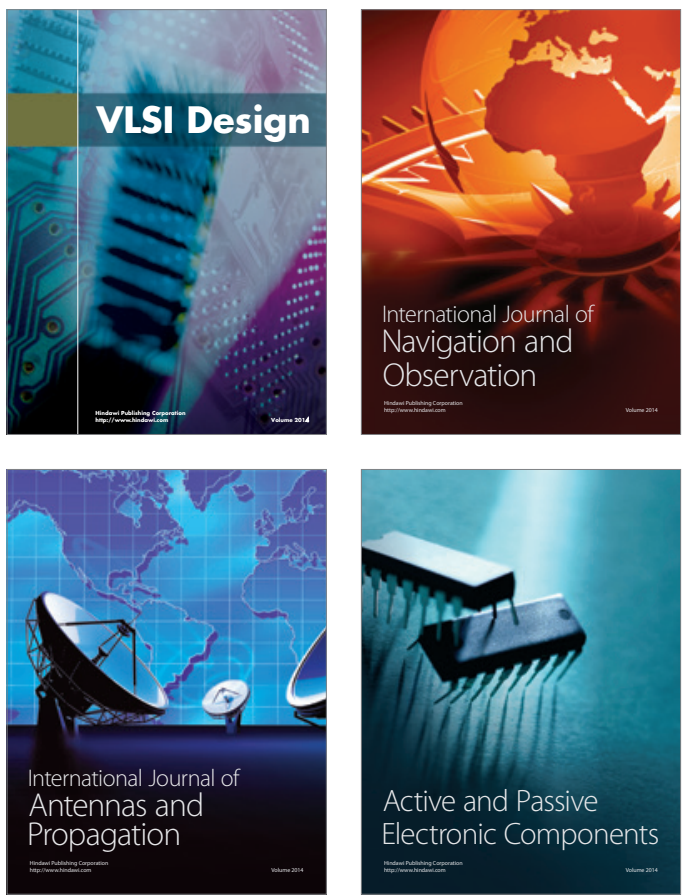
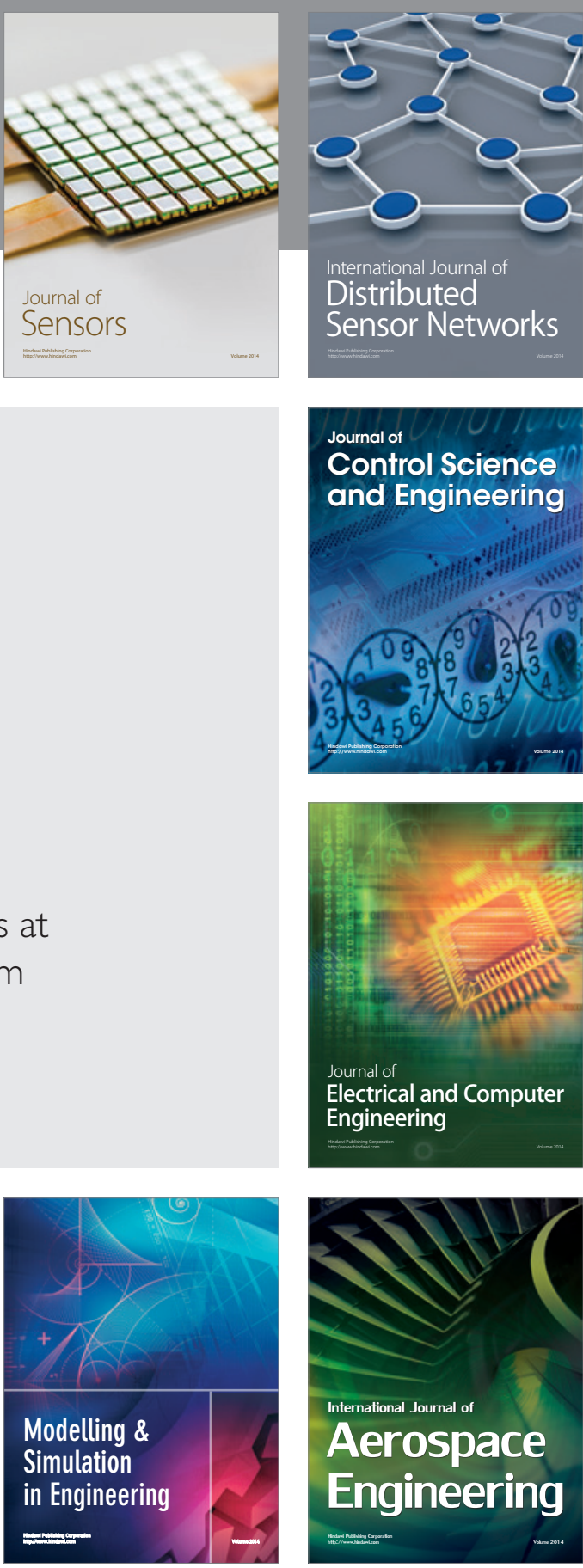

International Journal of

Distributed

Sensor Networks

Journal of

Control Science

and Engineering
